$$
R_{0}=\frac{1}{4} \log p_{1}-\log p_{2}^{2}{ }^{2} \sim \frac{1}{4} \frac{\left(p_{1}-p_{2}\right)^{2}}{p_{2}{ }^{2}}
$$

und das stimmt für kleines $R$ bis auf einen Fehler von der Größenordnung $R^{2}$ mit dem exakten Wert

überein.

$$
R=\frac{\left(p_{1}-p_{2}\right)^{2}}{\left(p_{1}+p_{2}\right)^{2}}
$$

Es gibt eine einfache anschauliche Deutung der Beziehung (15). Man erhält sie nämlich unmittelbar, wenn man den Potentialverlauf durch eine Treppenkurve approximiert und sowohl die Schwächung des primären Strahls als auch die mehrfache Reflexion vernachlässigt. Die Funktion $p(x)$ sei also eine Treppenkurve, die an den Stellen $x_{n}$ sehr kleine Sprünge $p_{n}$ macht. Gegen diese Potentialtreppe läuft von links eine Welle $e^{-i p_{1} x}$. An jeder Stufe wird eine rückläufige Welle mit der Amplitude 1/2 $\Delta p / p$ abgespalten. Die Intensität des reflektierten Strahles ist das Absolutquadrat der Summe aller Teilwellen an einem Punkte $x_{0}$ genügend weit rechts. Man muß nur be- rücksichtigen, daß diese Wellen untereinander Phasendifferenzen haben, die gleich dem doppelten Lichtweg von $x_{0}$ bis $x_{n}$ sind. Dieser ist gleich

$$
S\left(x_{0}, x_{n}\right)=\int_{x_{0}}^{x_{n}} p(x) d x
$$

und folglich ist

$$
R=\mid \sum_{n} \frac{1}{2} \frac{\Delta p_{n}}{p_{n}} \exp \left(-2 i S\left(x_{0}, x_{n}\right)\right) .
$$

Geht man zur Grenze sehr vieler sehr kleiner Sprünge über, so konvergiert die Summe bis auf einen belanglosen Faktor vom Betrage 1 gegen das Integral in (15). Diese Ableitung ist deshalb wesentlich, weil sie zu dem physikalisch befriedigenden Ergebnis führt, daß es keine Rolle spielt, ob $p(x)$ analytisch ist oder nicht, und daß eine analytische Funktion und eine Treppenfunktion denselben Reflexionskoeffizienten liefern, wenn sie nur hinreichend gut im Gesamtverlauf übereinstimmen.

\title{
Zur Genauigkeitssteigerung optischer Messungen durch Minimumstrahlkennzeichnung
}

\author{
Von Hans Wolter \\ Aus dem Institut für Experimentalphysik der Universität Kiel \\ (Z. Naturforschg. 5 a, 139-143 [1950]; eingegangen am 5. April 1949)
}

\begin{abstract}
Die prinzipielle Unschärfe eines Lichtstrahls kann überwunden werden, wenn auf die Energiekonzentration verzichtet wird. Eine Ebene im Raum kann durch geeignete Minima oder schwache Maxima prinzipiell beliebig genau gekennzeichnet werden; praktisch bedingen Störlicht (Streulicht, Inkohärenz u. a.) oder Mängel der Optik die Grenze. Eine Genauigkeitssteigerung um den Faktor 25 gelingt mit einfachen Mitteln.

Die Anwendung beschränkt sich nicht auf die Kennzeichnung einer Ebene oder unmittelbar verwandte Probleme (Strahlversetzung bei Totalreflexion, Lichtzeigergeräte u. ä.); die Minimumstrahlkennzeichnung führt auch zu einer Verbesserung der Schlierenverfahren und ist auf spektroskopische und mikroskopische Untersuchungen anwendbar. Ferner kann man den „Weg des Lichtes“ als geometrischen Ort der Minima definieren und so diesem Begriff einen experimentell greifbaren und theoretisch streng erfaßbaren Sinn geben.
\end{abstract}

\section{Die Unschärfe}

$\mathrm{I}^{\mathrm{n}}$ $\mathrm{n}$ der Wellenoptik bezeichnet man mit dem Worte „,Strahl“ ein Wellenbündel. Dies habe in einem seiner Querschnitte die flächenhafte Ausdehnung $\delta \dot{f}=\delta x \delta y$ und die Winkelstreuung $\delta \omega=\delta \alpha_{, r} \delta \alpha_{y j}$. Die Unschärfebedingung

$$
\partial x \delta \imath_{x} \geq i ; \quad \delta y \delta r_{y} \geq i,
$$

die der Heisenbergschen analog ist, zeigt die Unmöglichkeit, Winkelstreuung und Strahlbreite zu- gleich beliebig klein zu machen. Gleichung (1) gilt nur für kleine Winkel; allgemein benutzt man zweckmäßig die „Richtvariable“

$$
\gamma=\frac{\sin u}{i} .
$$

Mit ihr lautet die Unschärfebedingung

$$
\text { o) } x \partial y \geq 1 \text {. }
$$

Die reine Zahl $\delta x \delta \gamma$ benutzen wir als quantitatives Maß der „Unschärfe“, das Reziproke bezeichnen wir als „Schärfe“ g. 
Die für jede Strahlung gültige Unschärfebedingung hat speziell in der Optik die bekannten Auswirkungen auf das Auflösungsvermögen abbildender optischer Instrumente. Bei der Messung von Strahlversetzungen, wozu in der nachfolgenden Arbeit ein Beispiel behandelt wird, und bei der Messung von Strahlschwenkungen - z. B. bei Lichtzeigergeräten wie Spiegelgalvanometern - begrenzt sie die Meßgenauigkeit.

2. Der Zwang einer endlichen Durchlaßöffnung auf ein Wellenbündel

Die Unschärfegrenze 1 läßt sich, wie wir zeigen wollen, unterschreiten, wenn auf die Energiekonzentration des Strahles verzichtet wird. Da die Abbesche Sinusbedingung Invarianz der Strahlunschärfe $\delta x \delta \gamma$ gegen Abbildung mit idealen Linsen, Spiegeln, Prismen usw. bedeutet, genügt die spezielle Betrachtung einer ebenen Durchlaßöffnung der Breite $\delta x$, auf die eine ebene Welle der Amplitude $A$ senkrecht auffällt. Wir wollen die Amplitude und Phase des Lichtes in der Durchlaßöffnung durch geeignete Mittel (Platten u. ä.) beeinflussen und aus der einfallenden Welle der Amplitude $A$ die Feldstärkenverteilung $A B(x)$ in der Öffnung herstellen. Für die Belegungsfunktion $B(x)$ gilt natürlich $|B(x)| \leqq 1$. Wir suchen die Belegungsfunktion, mit der die Richtcharakteristik

$$
\begin{aligned}
& E\left(i^{\prime}\right)=A \int_{-\delta_{2}}^{+\frac{\delta x}{2}} B(x) \exp (-2 \cdot i ; x) d x \\
& =A \int B(x) \sum_{n=0}^{\infty} \frac{(-2 . \tau i \gamma x)^{n}}{n !} d x=A \sum_{n=0}^{\infty} c_{n} \gamma^{n} \\
& c_{n}=\frac{(-2.7 i)^{n}}{n !} \int_{-\frac{\delta x}{2}}^{+\frac{\delta x}{2}} x^{n} B(x) d x
\end{aligned}
$$

zur Strahlkennzeichnung besonders geeignet wird.

Die äußerste Energiekonzentration auf die Richtung $\gamma=0$ bewirkt die Belegung $B(x)=1$. Das Hauptmaximum des dann entstehenden Fraunhoferschen Beugungsbildes hat bekanntlich die Halbwertsbreite $\delta \gamma \approx 1 / \delta x$ und also die Schärfe 1 . Bei dem Suchen nach einer Vergrößerung der Schärfe stoßen wir zunächst auf eine Schranke, die den Entwicklungskoeffizienten $c_{11}$ durch die endliche Breitc $\delta . i$ der Durchlaßöffnung aufgezwungen wird

$$
c_{n} \leq \frac{.^{n}}{(n+1) !}\left({ }^{n} x\right)^{n+1} \text {. }
$$

Die Krümmung und die Steilheit $d|E(\gamma)| / d \gamma$ im Richtdiagramm

$E\left(\gamma^{\prime}\right)$

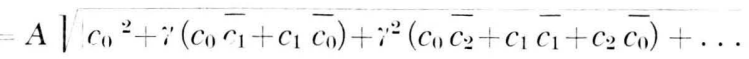

sind daher ebenfalls beschränkt. $\left(\bar{c}_{r}\right.$, konjugiert komplex $\mathrm{zu} c_{\nu}$. )

\section{Die Maximumverschärfung}

Insbesondere kann die Krümmung des Richtdiagramms $|E(\gamma)|$ an einem Maximum keinesfalls über den mit der trivialen Belegung $B(x)-1$ bereits erreichten Betrag $A{ }_{3}^{7^{2}}(\delta x)^{3}$ hinaus gesteigert werden.

Wir betrachten im Augenblick um der größeren Anschaulichkeit willen hier zunächst speziell reelles $E(\gamma)$; dann kann offenbar die Halbwertsbreite eines Maximums, dessen Krümmung bereits den maximalen Wert hat, nur noch dadurch verkleinert werden, daß die gesamte Kurve in der Umgebung des betrachteten Maximums stärker unter die Null-Linie gesenkt wird. Geht dabei die Feldstärke im Maximum auf den Bruchteil $m<1$, so fällt die Halbwertsbreite auf den Bruchteil $7 m$.

Hieraus folgt, und Verf. bewies es an anderer Stelle allgemein für komplexes $E(\gamma)$, daß durch geeignete Maxima eine prinzipiell beliebige Vergrößerung der Strahlschärfe $g=(\delta x \delta \gamma)^{-1}$ möglich ist, aber zwangsläufig durch Verlängerung der Belichtungszeit (Ausleuchtung der Öffnung konstant) um den Faktor

$$
\tau \approx 1 / m^{2} \approx g^{4}
$$

erkauft werden muß. Ist $I_{\mathrm{s}}$ das Verhältnis der Streulichtintensität an $\gamma=0$ zur Intensität des Maximums, das dort für $B(x)-1$ vorhanden wäre, so kann die Schärfe höchstens auf

$$
g=0,7 / 1 / I_{\mathrm{s}}
$$

(praktisch selten über 6 hinaus) gesteigert werden. Der hohe Exponent 4 in Gl. (7) erlaubt praktisch keine sehr wirksame Maximumverschärfung.

\section{Die Minimumstrahlkennzeichnung}

Die der Krümmung im Richtdiagramm von der endlichen Durchlaßöffnung aufgezwungene Schranke läßt nur scheinbar keine Hoffnung auf eine Verbesserung der im vorhergehenden Abschnitt erzielten Ergebnisse. Den Ausweg zeigt die Singularität der 
Wurzelfunktion in Gl. (6). Für $c_{0}=0$ ist $|E(\gamma)|$ $=A\left|c_{1} \gamma\right|+\ldots$, und an $\gamma=0$ liegt dann eine lineare Nullstelle; das Richtdiagramm hat eine Spitze und die Krümmung existiert dort nicht. Liegt ein wenig Streulicht vor, so ist die Krümmung jedenfalls sehr groß. Die Doppelwertsbreite ist klein und prinzipiell unbeschränkt.

Auf die Doppelwertsbreite kommt es bei einem Minimum an, ebenso wie bei einem Maximum auf die Halbwertsbreite. Denn die Lichtempfindung des Auges entspricht dem Logarithmus der Intensität (oder der Feldstärke), und die Breite eines Graubereichs auf einer Photographie ist der logarithmischen Ableitung des Feldstärkenverlaufs umgekehrt proportional. Die Brauchbarkeit einer Richtcharakteristik zur Kennzeichnung einer Ebene im Raum wird daher zweckmäßig am $\log |E(\gamma)|$ beurteilt, statt unmittelbar am Intensitätsverlauf selbst ${ }^{1}$. Da nun der $\lim _{E(\gamma) \rightarrow 0} \log |E(\gamma)| \rightarrow-\infty$, verläuft $\log |E(\gamma)|$ in der Umgebung der Nullstellen sehr steil, und der Vorteil der Nullstellen wird evident. Durch die Steilheit dieses Verlaufs wird die photographische Graubreite diktiert, die Verf. an anderer Stelle unmittelbar als Maß für die Güte der verschiedenen Strahlkennzeichnungsmethoden benutzt hat. Hier mag um der Kürze willen die Doppelwertsbreite im Feldstärkenverlauf zur Charakterisierung genügen; sie werde bei einem Minimum mit $\delta \gamma$ bezeichnet, und mit diesem $\delta \gamma$ seien die Begriffe Unschärfe $\delta x \delta \gamma$ und Schärfe $g=(\delta x \delta \gamma)^{-1}$, die bisher nur für Maxima definiert waren, auf Minima erweitert.

Die einfachste Realisierung linearer Nullstellen vom Typ $|E(\gamma)|=A\left|c_{1} x\right|+\ldots$ kann durch zwei enge Spalte des Abstandes $\delta x$ geschehen oder allgemeiner durch zwei kohärente spaltförmige Lichtquellen, die in bekannter Weise mittels Biprismas oder Fresnelschen Doppelspiegels aus einer Lichtquelle

1 Daher ist die landläufige Vorstellung, daß Maxima und Minima im Intensitätsverlauf gleiche "Breite“ hätten, zwar bei entsprechender Definition der „Breite“ richtig; doch trifft sie nicht das Wesentliche. An jener Vorstellung mag es auch liegen, daß der Unterschied der Maxima und der Minima in der Optik in seiner Bedeutung weder voll erkannt noch konsequent ausgenutzt $\mathrm{zu}$ werden pflegt. Der einzige Hinweis in der Literatur auf diesen Unterschied scheint eine Fußnote bei Is ing, Ann. Physik 14, 769 [1932], zu sein: „Wenn dagegen das Bild“ (gemeint ist das oben behandelte Fraunhofersche Beugungsbild für $B(x) \quad 1$;) ,in einem Okular aufgefangen wird, sieht man auch die ersten seitlichen Maxima, und die zwischenliegenden Minima stehen als verhältnismäßig scharfe, dunkle Referenzlinien hervor, die eine genauere Ablesung gestatten“. Unsymmetrische und flache Flanken setzen jene Minima freilich stark in Nachteil gegenüber dem mit der Belegung (9) herstellbaren (s. u.!). hergestellt werden können. Alle diese Verfahren mögen als Verdopplungsverfahren bezeichnet werden. Gemeinsam ist ihnen eine Richtcharakteristik, in der Maxima und lineare Minima abwechseln. Die Nullebenen (Ebenen, auf denen $E=0$ ist) divergieren dabei zunächst; mit einer Sammellinse kann man aber leicht auch ein System paralleler Nullebenen daraus erzeugen, das nun durch Interferenz zweier ebener Wellen entsteht. Da das Schicksal zweier ebener Wellen auch in einer komplizierten Apparatur oft noch streng zu berechnen ist, kann die Theorie den Weg eines so entstehenden Minimums auch in solchen Fällen streng verfolgen, in denen das für einen Energiestrahl $(B(x)=1)$ mathematisch nicht mehr gelingt. Wir werden davon in der nachfolgenden Arbeit Gebrauch machen.

Zugleich ist das Minimum um Größenordnungen genauer zu vermessen. In dieser Hinsicht stellt allerdings das Verdoppelungsverfahren noch nicht das Optimum dar. Denn die mit einem linearen Minimum praktisch erreichbare Strahlschärfe wird entweder durch Streulicht oder durch die unzureichende Empfindlichkeit der Netzhaut bzw. der Photoschicht und im Zusammenhang damit schließlich durch die Flankensteilheit $c_{1}$ der Feldstärke zu seiten des Minimums bestimmt. Diese nimmt bei gegebenem $\delta x$ ihren nach (5) größtmöglichen Betrag an für die optimale Belegung

$$
B(x)=\left\{\begin{array}{l}
+1 \text { für } 0 \leq x<\frac{\delta x}{2} \\
-1 \text { für }-\frac{\partial x}{2} \leq x<0 .
\end{array}\right.
$$

Der Nachweis dafür, daß unter allen möglichen diese Belegung im wesentlichen (d. h. abgesehen von einem uninteressanten konstanten Phasenfaktor oder von Abänderungen der Funktion an einer Argumentmenge vom Maß 0) die einzige ist, die zu einer so großen Flankensteilheit führt, ist vom Verf. an anderer Stelle erbracht worden. Diese Belegung wurde realisiert durch eine Phasenplatte oder einen Phasenspiegel oder einen Phasenschieber, die das Licht der einen Hälfte in der Durchlaßöffnung gegen das der anderen um $180^{\circ}$ in Phase drehten ${ }^{2}$. Abb. $1^{*}$ zeigt unten die photographische Aufnahme des zugehörigen Richtdiagramms in einer sehr entfernten Ebene. Das scharfe Minimum hat eine Doppelwertsbreite, die nur $1 / 26$ der Halbwertsbreite eines üblichen Maximumstrahles (oben) mit $B(x) \quad 1$ ist. Die Belichtungszeit betrug unten $50 \mathrm{sec}$, oben etwa $0,3 \mathrm{sec}$.

2 Näheres hierzu erscheint in Kürze in den Ann. Physik. * s. Tafel S. 144 b. 
Solange mangelnde Helligkeit zu seiten des Minimums die Strahlschärfe begrenzt (infolge nicht hinreichender Annäherung der Graubereiche an das Minimum), besteht zwischen der Verlängerung der Belichtungszeit $\tau$ und der Strahlschärfe $g$ die Beziehung

$$
T \approx 0,3 g^{2} .
$$

Wenn die Belichtungszeit so lang gewählt werden kann, daß eine weitere Verlängerung wegen des Störlichtes keine Verbesserung mehr gibt, so gilt

$$
r \approx 0,6 g^{2} ; \quad g=0,6 / / \overline{I_{\mathrm{s}}}
$$

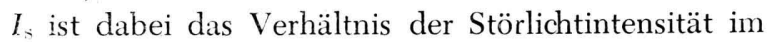
Minimum zur Intensität des Maximums bei $B(x) \quad 1$.

Die Brauchbarkeit der Minimumstrahlkennzeichnung und ihre Überlegenheit über die Maximumverschärfung wird durch den kleineren Exponenten (2 statt 4) in Gl. (10) entschieden. Die Verschärfung wird. hier mit einer kürzeren Belichtungszeit erreicht, vor allem aber läßt die Minimumstrahlkennzeichnung bei einer relativen Störlichtintensität von $0,5 \%$ noch eine Strahlschärfe $27 \mathrm{zu}$, während die Maximumverschärfung damit höchstens zu 5 führen würde. Die entscheidende Ursache für diesen Unterschied ist die Linearität des Minimums. Auch quadratische Minima vom Typ $E(\gamma)=A c_{2} \gamma^{2}+\ldots$ haben prinzipiell die Doppelwertsbreite 0 und lassen daher beliebige Strahlverschärfung zu. Bezüglich der Belichtungszeit und gegenüber dem Streulicht verhalten sie sich ähnlich wie die Maxima. Während es nun bei den Maxima naturgemäß nur quadratische gibt, gibt es unter den Minima auch lineare.

Drei Ursachen bestehen also für den Erfolg der Minimumstrahlkennzeichnung:

a) Die Bedeutung der Funktion $\log |E(\gamma)|$ für die visuelle oder photographische Beobachtung. Relative Intensitäten und daher Halbwertsbreiten bzw. DopFeiwertsbreiten sind maßgebend für die Strahlschärfe. (Davon profitieren alle Minima, auch quadratische usw., ja letzten Endes auch die schwachen Maxima.)

b) Die Linearität der Minima vom $\operatorname{Typ} E(\gamma)=c_{1} \gamma$ $+\ldots$ (Sie sorgt für den relativ geringen Belichtungsaufwand bzw. für den relativ kleinen Einfluß des Störlichtes im Vergleich mit den quadratischen Minima oder den notwendig quadratischen Maxima. Diesen Vorteil bieten bereits die ebenfalls linearen Minima des Fresnelschen Spiegelversuchs.) c) Die optimale Flankensteilheit des mit der Belegung (9) hergestellten Richtdiagramms. (Sie sichert die Ưberlegenheit gegenüber allen anderen Verfahren; sogar für alle anderen linearen Minima gilt beispielsweise die zweite Gleichung (11) mit einer kleineren Zahl im Zähler.)

\section{Kohärenztoleranzen}

Die endliche Durchlaßöffnung der Breite $\delta x$, die wir eingangs betrachteten, wird durch einen Kollimator beleuchtet, in dessen Brennebene ein leuchtender Spalt der Breite $\delta x_{*}$ und der ausgenützten Apertur $\delta \gamma_{*}$ (im Maßstab der Richtvariablen) liegt. Strenge Kohärenz wäre nur bei $\delta x_{*} \rightarrow 0$ vorhanden; doch ist sie wegen notwendig endlicher Flächenhelligkeit nicht möglich; sie ist auch nicht erforderlich. Die zulässige Spaltbreite wird in der Literatur auf Grund des Verdetschen Satzes ${ }^{3}$ beurteilt, der in unseren Bezeichnungen der Forderung $\delta x_{*} \delta \gamma_{*} \ll 2$ äquivalent ist. Die in der Literatur gegebenen Begründungen wären aber nur dann stichhaltig, wenn die „experimentelle Feststellbarkeit" tatsächlich durch die Unschärfebedingung $\delta x \delta \gamma \geqq 1$ allgemein begrenzt wäre. Wie wir zeigten, trifft das nicht zu; daher ist die übliche Kohärenzbedingung durch die folgende zu ersetzen: Die „laterale Inkohärenz der Lichtquelle" $\delta x_{*} \sigma \gamma_{*}$ muß klein gegenüber der Unschärfe der benutzten Strahlkennzeichnung sein. Die Begründung ergibt sich unmittelbar aus der oben (Abschnitt 2) genannten Invarianz des Produktes $\delta x_{*} \delta \gamma_{*}$ gegen ideale Abbildungen.

Entsprechend ist die übliche Tiefenkohärenzbedingung zu verschärfen zu der Aussage, daß die Breite des benutzten Spektralbereichs $\delta \lambda$ das den Strahl kennzeichnende Gebilde (z. B. das Minimum) der Schärfe $g$ nicht merklich verbreitert, wenn

$$
\delta \lambda / \lambda \ll 1 / n g
$$

ist; darin ist $n$ der Gangunterschied der beiden interferierenden Bündel, gemessen in Wellenlängen. Bei unserer Belegung (9) ist $n=0,5$.

\section{Subtilität eines Effektes}

Die Minimumstrahlkennzeichnung kann dort mit Nutzen angewandt werden, wo kleine Ablenkungen des Lichtes oder Strahlversetzungen o. ä. gemessen werden sollen. $\mathrm{Ob}$ sie dazu im Einzelfall erforderlich ist, wird nicht durch die Kleinheit der zu messenden Größe allein bestimmt. Wird z. B. die Messung eines kleinen Winkels $\Delta \alpha$ verlangt, so ist zugleich die verfügbare Durchlaßöffnung und ihre Breite $\Delta x$ entscheidend.

3 M. Berek, Z. Physik 53, 483 [1929]; K. W. Koh lrausch, Ausgewählte Kapitel aus der Physik, 2. Teil, Optik, Wien 1948 u. a. 
Allgemein erweist es sich als zweckmäßig, die "Subtilität" eines Effektes quantitativ durch die Größe

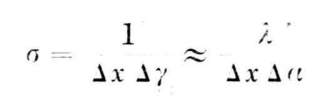

begrifflich zu fassen. Ein Effekt kann also nur mit einer Strahlkennzeichnung hinreichend großer Schärfe $g \geqq 0$ gemessen werden. $g / \sigma=\mu$ heiße „Meßbarkeit“; ist sie 1, so kann der Effekt mit der betreffen- den Strahlkennzeichnung noch auf etwa 10 bis $20 \%$ genau gemessen werden. Die Nachweisgrenze liegt bei etwa $\mu=0,2$. Wegen der Invarianz von $g, \sigma$ und $\mu$ gegen Abbildungen helfen diese Begriffe in verwickelten Fällen prinzipielle Fragen nach der M€ßbarkeit subtiler Effekte unmittelbar beantworten.

Hrn. Prof. Dr. Lochte-Holtgreven und Hrn. Prof. Dr. Uns öld, die die Wiederaufnahme dieser Arbeit ermöglichten und sie in jeder Weise unterstützten, möchte der Verf. an dieser Stelle danken.

\title{
Untersuchungen zur Strahlversetzung bei Totalreflexion des Lichtes mit der Methode der Minimumstrahlkennzeichnung*
}

\author{
Von Hans Wolter \\ Aus dem Institut für Experimentalphysik der Universität Kiel \\ (Z. Naturforschg. 5 a, 143-153 [1950]; eingegangen am 5. April 1949)
}

\begin{abstract}
Mit der Methode der Minimumstrahlkennzeichnung wurde die Strahlversetzung bei Totalreflexion gemessen. Experimente und Theorie stimmen hier auch bezüglich der starken Polarisationsabhängigkeit überein, im Gegensatz zu dem früheren Befund von G o o s und Hän che n ${ }^{1}$. Messungen mit einer verbesserten Maximummethode zeigten ebenfalls (freilich ungenauer) diєse Polarisationsabhängigkeit. Strahlversetzung wurde auch bei Reflexion an absorbierenden Medien festgestellt; sie wechselt hier meist mit der Polarisationsrichtung ihr Vorzeichen. Eine ausführliche Zusammenfassung befindet sich am Schluß.
\end{abstract}

\section{I.}

G oos und Hänchen ${ }^{1}$ fanden folgenden Effekt, für den hier die Bezeichnung Goos-HänchenEffekt vorgeschlagen sei. Ein durch zwei hintereinandergestellte Spalten ausgeblendeter Lichtstrahl wird bei Totalreflexion nicht an der Stelle der Grenze Glas-Luft, auf die er auftritt, reflektiert, sondern um eine kleine Strecke so parallel verschoben, als ob er einen Umweg durch das dünnere Medium gemacht hätte. Der Effekt liegt in der Größenordnung einer Wellenlänge; Goos und Hänchen vergrößerten ihn durch 34-fache Wiederholung der Reflexion und konnten die Verschiebung nach ihren eigenen Angaben auf $2 \%$ genau messen. Innerhalb dieser Grenzen fanden sie keine Abhängigkeit von der Polarisation.

Das widersprach unveröffentlichten Rechnungen des Verf., die zur Erklärung einer ähnlichen Versetzung bei elektromagnetischen Längstwellen dienten und infolge ihres sehr allgemeinen Ansatzes leicht auf die Totalreflexion spezialisiert werden konnten.

1 F. Go os u. H. Hänchen, Ann. Physik (6) 1, 333 [1947].

2 K. A rtm a n n, Ann. Physik (6) 2, 87 [1948].
Sie werden hier in II den Ausgangspunkt dieser Arbeit bilden. Die darauf aufbauenden experimentellen und theoretischen Untersuchungen haben auf zwei Fragen eine neue Antwort gegeben. Die erste behandelt die Abhängigkeit der Strahlversetzung in dichteren Medium vom Einfallswinkel und von der Polarisation; sie ist Gegenstand dieser Arbeit. Die zweite fragt nach den Vorgängen im dünneren Medium und an der Grenzfläche - also insbesondere nach dem "Umweg des Lichtes" — und wird in einer später zu veröffentlichenden Arbeit beantwortet.

Zur Klärung der ersten Frage trug inzwischen Artmann² wesentlich bei, der eine Theorie des Goos-Hänchen-Effekts gab (unter genauer Nachbildung der bei Goos und Hänchen herrschenden Verhältnisse) und eine Polarisationsabhängigkeit ausrechnete. Nach einer Betrachtung über mögliche Fehlerquellen, die ausgeschlossen werden konnten, vermutete Artmann aber schließlich die Ursache der Diskrepanz zwischen Theorie und Experiment in seiner Näherung, die statt der mathematisch zu schwie-

* Vorgetragen auf der Tagung der Deutschen Physikalischen Gesellschaft in Hamburg am 23. April 1949. 